\title{
Un Asellide interstitiel du bassin de l'Ebre (Espagne): Proasellus lescherae n.sp. (Crustacea, Isopoda, A sellota).
}

par

\author{
Jean-Paul HENRY et Guy MAGNIEZ*
}

An interstitial Asellid from Ebro basin (Spain): Proasellus lescherae n.sp., (Crustacea, Isopoda, Asellota).

Proasellus lescherae n.sp. is a little, interstitial species, living in the underflow of Rio Guadalope Basin (a tributary of Ebro River). It is related to the water-slaters of the underground waters of the Pyreneo-cantabrigian region (the phyletic line of Proasellus spelaeus $\mathrm{R}$ acovitza). The mean size of the reproductive females is very small $(2,9 \mathrm{~mm})$ and the brood contains only $6-10$ eggs. Nevertheless, the size of the eggs and intramarsupial larval stages are the same as in epigean or hypogean species of the genus, which reach a larger adult size.

Origine: Ce nouvel Asellide apigmenté et anophtalme a été capturé par Mesdemoiselles N. Gourbault et F. Lescher-Moutoué et Monsieur R. Rouch, du Laboratoire Souterrain du C.N.R.S. à Mo'llis (Ariège), au cours de leurs prospections de la faune des eaux souterraines d'Espagne, en septembre 1976. Nous les remercions très vivement de leur confiance et nous dédions cette espèce à Mademoiselle F. Lescher-Moutoué.

Situation des stations: Au nombre de 4, elles se situent sur le Rio Guadalope, affluent de rive droite de l'Ebre et sur un affluent de rive droite du Rio Guadalope: le Rio Bergantes (fig. 1).

1. Station-type: Sous-écoulement du cours supérieur du Rio Guadalope à Villaroya de los Pinares, Prov. Teruel (station 46 des prospecteurs). Elle a été choisie par suite de la plus forte taille des individus capturés, sans doute en raison de la granulométrie plus favorable des alluvions du lit de la rivière: $41 \hat{\delta}$ adultes de 2,6 à $3,9 \mathrm{~mm}+12$ adultes de 2,4 à $4,4 \mathrm{~mm}$ (dont 2 à marsupium vide, de 3,7 et $4,4 \mathrm{~mm}$ ) + une vingtaine de jeunes de 1 à $2 \mathrm{~mm}$ et quelques larves

* Laboratoire de Biologie Animale et Générale, Université de Dijon, 6, bd Gabriel, 21100-Dijon, France. 

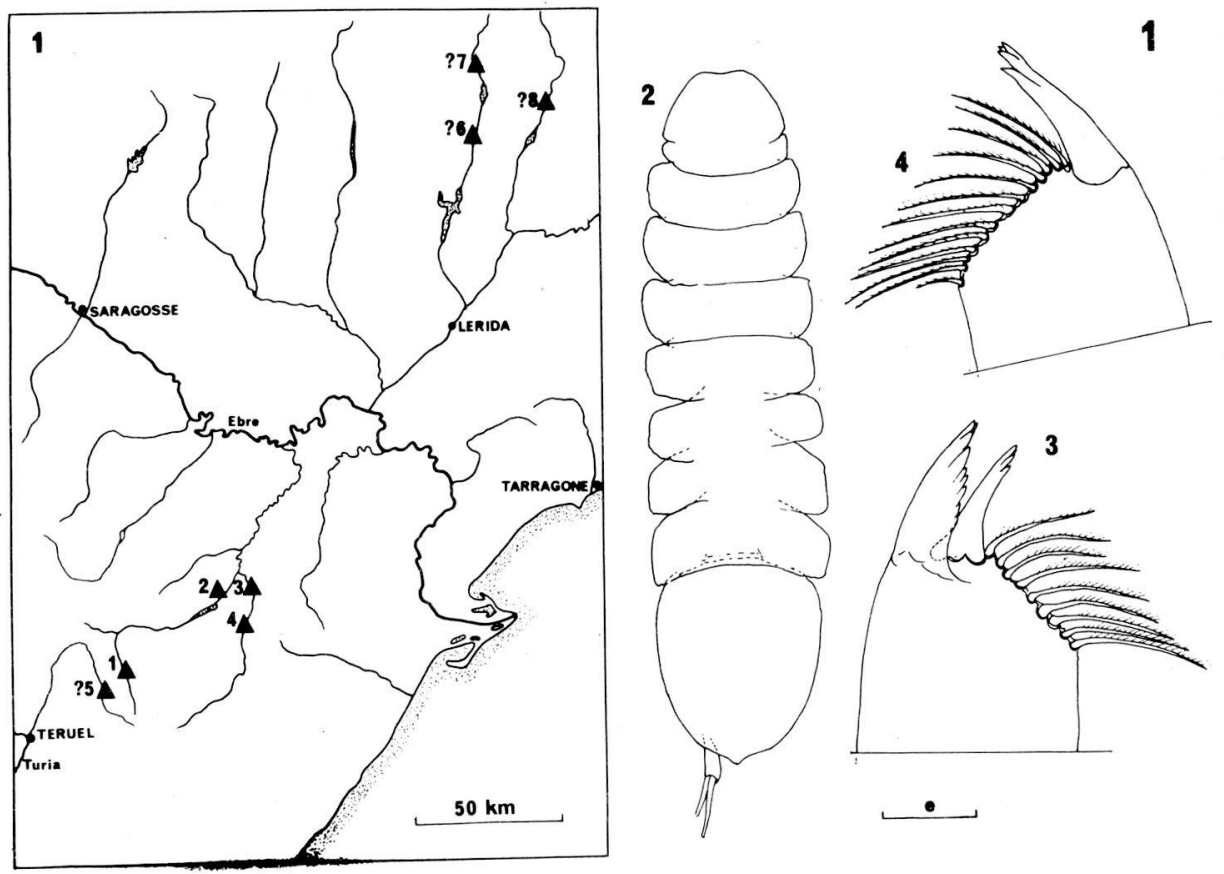

Fig. 1. Carte schématique du N.E. de l'Espagne: $\mathrm{I}=\mathrm{R}$ io Guadalope à Villeroya de los Pinares, $2=$ Rio Guadalope à Mas de las Matas, $3=$ Rio Bergantes à Aguaviva, $4=$ Rio Bergantes entre Ortélis et Zorita del Maestrazgo, 5 = Rio Alfambra à Allepuz, 6= Rio Noguera Ribagorzana entre Arén et Puente de Montañana, $7=$ Rio Noguera Ribagorzana à Pont de Suert, $8=$ Rio Noguera Pallaresa, défilé de Collegats.

Fig. 2. Habitus d'un $\widehat{O}$ de $3,75 \mathrm{~mm}$ de la station-type, $\mathrm{e}=500 \mu$.

Fig. 3. Partie distale de la mandibule gauche de l'allotype $q, \mathrm{e}=50 \mu$.

Fig. 4. Idem, mandibule droite, $\mathrm{e}=50 \mu$.

marsupiales de 0,8 à $0,9 \mathrm{~mm}$. (holotype: le $\delta \hat{~ d e ~} 3,9 \mathrm{~mm}$, allotype, la $q$ à marsupium vide de $4,4 \mathrm{~mm}$ ).

2. Sous-écoulement du cours moyen du Rio Guadalope à Mas de las Matas, Prov. Teruel (station 50 des prospecteurs): 13 $\delta$ adultes de 2,2 à $2,8 \mathrm{~mm}+15 q$ adultes au repos génital de 2,5 à $3,5 \mathrm{~mm}+4$ 文 marsupium vide de 2,8 à $3,1 \mathrm{~mm}+$ environ 70 jeunes et immatures de 1 à $2 \mathrm{~mm}$.

3. Sous-écoulement du Rio Bergantes à Aguaviva, Prov. Teruel (station 49 des prospecteurs): $74 \hat{\delta}$ adultes de 2 à $3,1 \mathrm{~mm}+53$ $q$ radultes au repos génital de 2 à $3,3 \mathrm{~mm}+22+$ adultes en intermue de reproduction, de 2,5 à $3,5 \mathrm{~mm}$ (taille moyenne: $2,93 \mathrm{~mm}$ ). Parmi elles, 5 sont ovigères: $2,5 \mathrm{~mm}$ ( 8 embryons), $2,7 \mathrm{~mm}$ ( 8 oeufs) $2,8 \mathrm{~mm}$ ( 6 embryons), $2,9 \mathrm{~mm}$ ( 7 embryons) et $3,5 \mathrm{~mm}$ ( 9 oeufs). Taille de l'oeuf: grand axe de $240 \mu$ environ et petit axe de $210 \mu$ environ. Le lot comporte encore 45 individus: quelques adultes fragmentés, des immatures de l'un et lautre sexe, des jeunes de sexe externe indifférencié, quelques larves. L'im- 
portance de l'activité reproductrice dans cette station est surprenante, compte tenu de la saison de la capture, la fin de l'été étant généralement la période où la reproduction passe par un minimum d'activité (Henry, 1976).

4. Sous-écoulement du Rio Bergantes, entre Ortélis et Zorita del Maestrazgo, Prov. Castellón (station 48 des prospecteurs): 11 î adultes de 1,9 à $2,7 \mathrm{~mm}+$ 6 ㅇ adultes de 2,1 à $3,4 \mathrm{~mm}$ (dont 19 ovigère de $2,9 \mathrm{~mm}$ avec 10 oeufs et $2 q$ à marsupium vide de 3 et $3,4 \mathrm{~mm}$ ) +2 jeunes de $1,8 \mathrm{~mm}$.

Remarque: Les prospections dans le sous-écoulement des rivières du Nord-Est de l'Espagne ont permis de noter la présence d'Aselles hypogés en d'autres points (stations notées 5, 6, 7 et 8 sur la carte, fig. 1). A chaque fois, il s'agissait de jeunes individus, non déterminables spécifiquement et nous n'en avons pas tenu compte pour la description de $P$. lescherae n.sp.:

5. Sous-écoulement du Rio Alfambra (affluent du Rio Turia, station 45 de prospecteurs) à Allepuz, Prov. Teruel.

6. Sous-écoulement du Rio Noguera Ribagorzana (stations 1, 2 et 3 des prospecteurs) entre Arén et Puente de Montañana, Prov. Lérida.

7. Sous-écoulement du même, près de Pont de Suert (Cl. Bou, leg. 1972) cf. Magniez, 1975.

8. Sous-écoulement du Rio Noguera Pallaresa, dans le défilé de Collegats, Prov. Lérida (Cl. Bou leg. 1972), cf. Magniez, 1975.

Caractères généraux: Comme l'indique la taille moyenne des $\bigcirc$ adultes en intermue parturielle $(2,9 \mathrm{~mm})$, il s'agit d'une espèce de petite taille. Les plus grands $\hat{o}$ capturés n'atteignent pas $4 \mathrm{~mm}$, tandis que les plus grandes $\$$ ne dépassent que de peu cette longueur. Les oeufs ayant des dimensions peu différentes de celles des espèces plus grandes, leur nombre (6-10) est très faible dans chaque ponte.

Le corps est modérément allongé (coefficient d'allongement de 3,8 seulement) (fig. 2). Le tégument est mince et souple et la chétotaxie générale (corps et appendices) très réduite.

Céphalon subtrapézoïdal à lobes postmandibulaires peu saillants. Coxopodites des péréiopodes I-VII débordant les marges latérales des péréionites et visibles en vue dorsale.

Pléotelson allongé ( $1,08 \mathrm{~mm}$ de long sur $0,88 \mathrm{~mm}$ de large chez un $\delta \hat{\mathrm{de}} 3,7 \mathrm{~mm}$ ).

Une paire de papilles génitales subcylindriques de $210-230 \mu$ de long et $35-40 \mu$ de diamètre insérées près de la marge caudale du dernier sternite thoracique du $\hat{o}$.

Largeurs respectives de la tête, des 7 péréionites et du pléotelson pour un de 3,7 mm: 0,67-0,81-0,88-0,91-0,90-0,96-0,97-0,95 et 0,88.

Pléonites I et II libres et étroits formant un pédoncule rétréci au pléotelson.

Appendices céphaliques: Antennules très courtes: $530 \mu$ pour un $\widehat{\delta}$ de $3,9 \mathrm{~mm}$

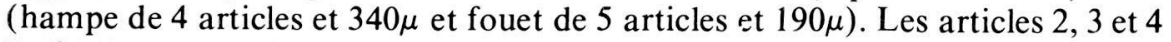
du fouet portent chacun une lame olfactive de $50 \mu$ de long. Le fouet ne comporte que 4 articles et 2 lames olfactives seulement chez les plus grandes + . 


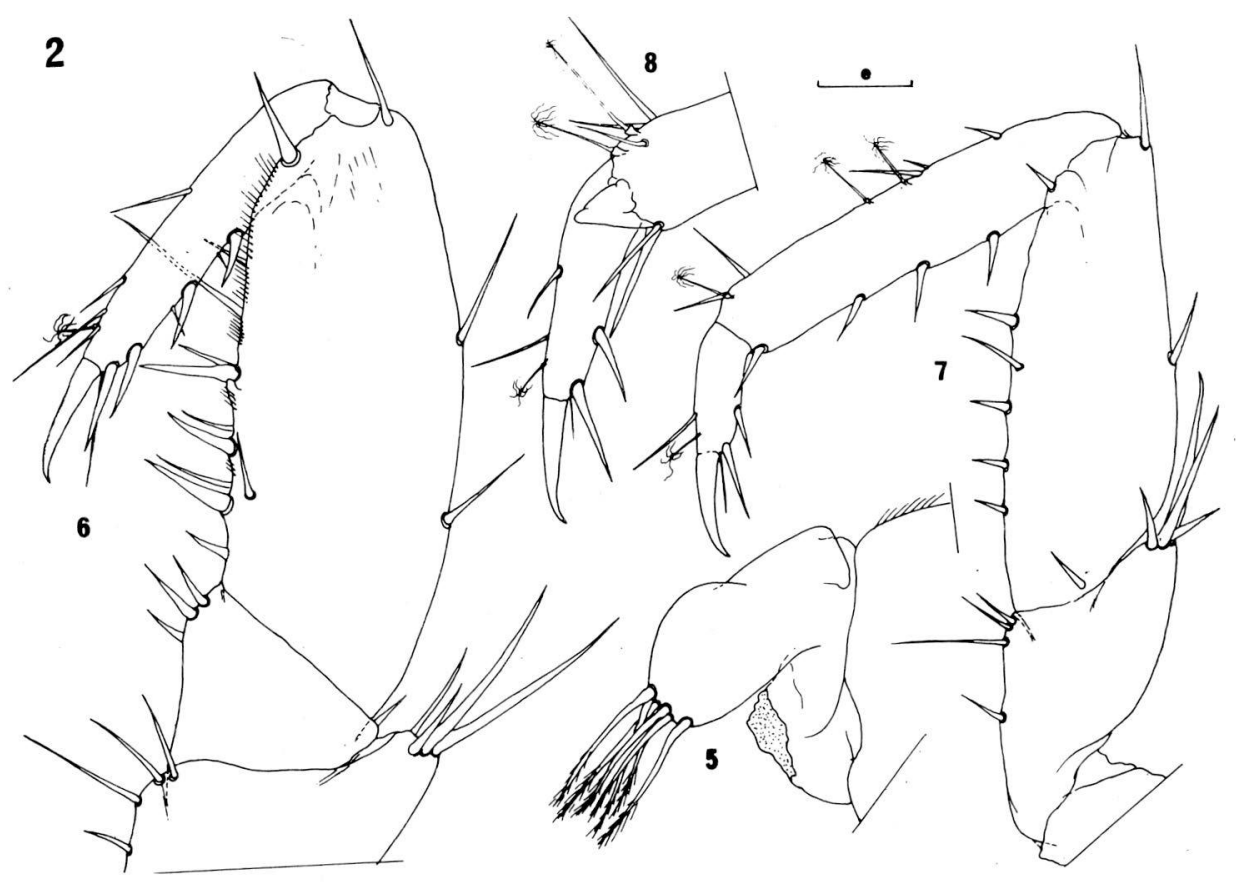

Fig. 5. Oostégite du maxillipède gauche de l'allotype ( $Q$ à poche incubatrice de $4,4 \mathrm{~mm}$ ), $\mathrm{e}=100 \mu$.

Fig. 6. Péréiopode I droit de la même, $\mathrm{e}=50 \mu$.

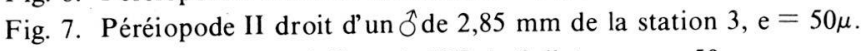

Fig. 8. Extrémité d'un péréiopode VII de l'allotype, $\mathrm{e}=50 \mu$.

Antennes longues et fragiles $(3,30 \mathrm{~mm}$ pour le ô de $3,9 \mathrm{~mm})$. Hampe de 6 articles et fouet de 35-40 au maximum.

Mandibules typiques avec parties incisives très dissymétriques (fig. 3, 4). Lacinia mobilis de la mandibule gauche avec 4 dents, suivie d'une rangée de 9 (13 à droite) tiges rameuses de 65-70 $\mu$. Lobe molaire allongé et garni distalement de très courtes tiges très serrées. Maxillules typiques avec lobe interne garni de 5 longues soies plumeuses ( 65 à $90 \mu$ de long); lobe externe avec 12 fortes tiges barbelées distales ( 35 à $45 \mu$ ) et une longue soie plumeuse submarginale distale externe.

Maxilles trilobées typiques: lobe externe avec une rangée de 23 lames falciformes pectinées intérieurement, décroissantes de 160 à $40 \mu$ de longueur vers l'intérieur; lobe médian avec 16 lames identiques aux précédentes et décroissant de 170 à $45 \mu$; lobe interne à double crête distale avec une trentaine de tiges polymorphes de 40 à $100 \mu$ environ.

Maxillipèdes: typiques d'Asellides; épipodite subquadrangulaire lamelleux et quasi glabre, endite avec rétinacle à 5 crochets; palpe normal à 5 articles. Chez 


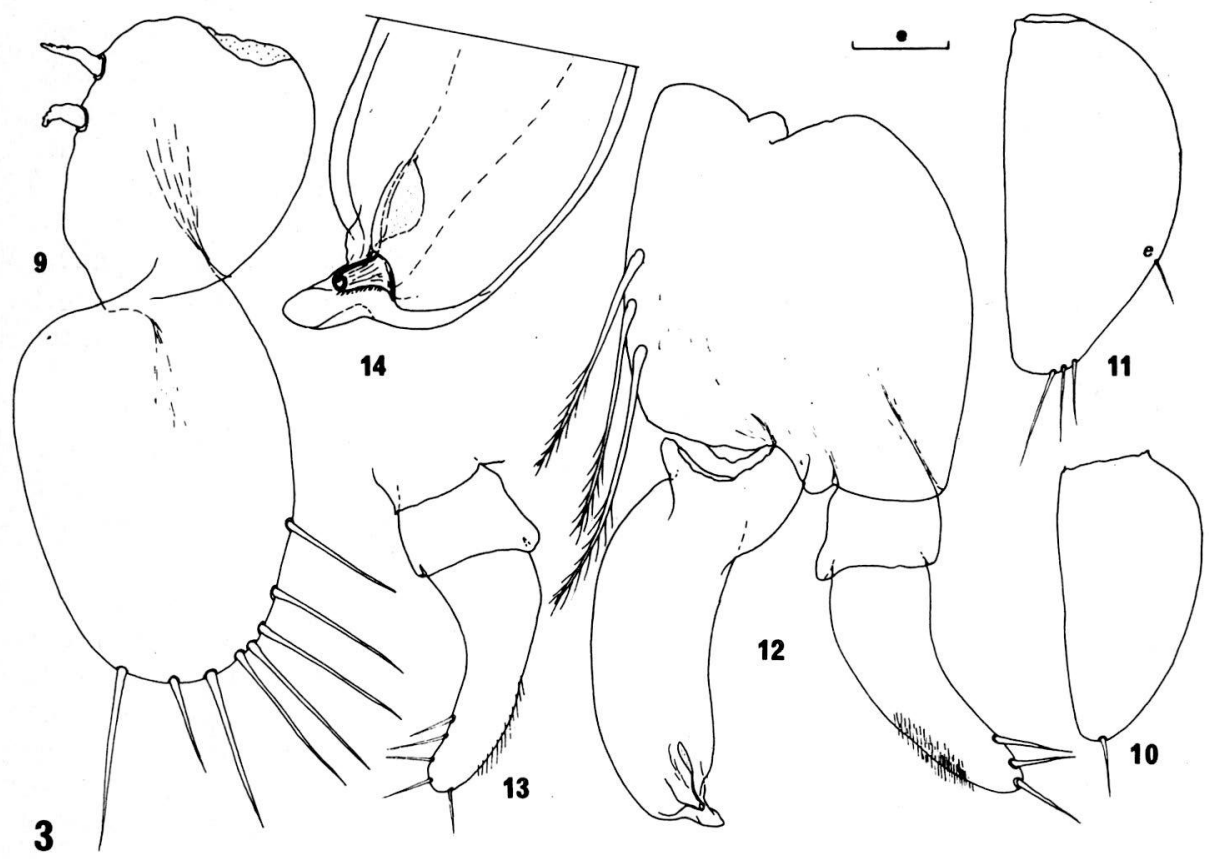

Fig. 9. Pléopode I gauche du type ( ôde $3,9 \mathrm{~mm}$ ), e $=50 \mu$.

Fig. 10. Pléopode II gauche d'une $Q$ de $3,6 \mathrm{~mm}$ de la station $1, \mathrm{e}=100 \mu$.

Fig. 11. Idem, de Pallotype, $\mathrm{e}=100 \mu$.

Fig. 12. Pléopode II gauche du type, $\mathrm{e}=50 \mu$.

Fig. 13. Exopodite du pléopode II droit, d'un ơde $3,75 \mathrm{~mm}$, de la station-type, e $=50 \mu$.

Fig. 14. Extrémité de Pendopodite du pléopode II droit du type, en vue sternale: apophyse tergale, goulot fendu et orifice afférent de la vésicule spermatique sont bien visibles, $\mathrm{e}=20 \mu$.

les $\oint$ à marsupium, le coxopodite porte un lobe sétigère (avec jusqu'à 7 soies plumeuses dirigées caudalement) transitoire (Wasserstrudelapparat) assurant le renouvellement de l'eau dans la poche incubatrice (fig. 5).

Péréiopodes I à VII: Grêles et fragiles, ils sont amputés au niveau de la partie proximale du basipodite chez la plupart des individus. Longueurs respectives des péréiopodes chez un ồ de 2,9 $\mathrm{mm}$ provenant d'Aguaviva: 0,93-1,13-1,171,14-1,32-1,53 et 1,65. Longueurs relatives au péréiopode II: 0,82-1,00-1,04$1,01-1,17-1,35$ et 1,46 . Les péréiopodes I sont presque aussi grêles que les suivants (fig. 6). Leur propodite est légèrement plus renflé chez le $\hat{\delta}$, ce dimorphisme sexuel est peu apparent. Le péréiopode II présente un carpopodite très légèrement renflé. Il est sans doute utilisé par l'animal pour se fixer sur les grains de sable, les péréiopodes I étant réservés aus maintien des particules nutritives (fig. 7). 
Le péréiopode IV, légèrement plus court que les II et III n'est pas transformé en crochet nuptial chez les $\delta$. Le dactylopodite des péréiopodes II à VII porte une seule épine sternale insérée juste sous l'ongle et très longue. Chez les plus grands individus capturés, il existe une seconde épine sternale plus proximale. Elle semble apparaître par priorité sur les péréiopodes II et VII (fig. 8).

Pléopodes I $\delta$ : Protopodite à peine plus long que large avec un rétinacle asymétrique ( 2 crochets d'un côté et 1 crochet de l'autre). Exopodite très court (fig. 9) avec jusqu'à 9 tiges marginales distales et externes, relativement courtes et toutes lisses.

Pléopodes II $q$ : Subtriangulaires et allongés, ils ne portent, chez les petites $q$ adultes qu' une seule tige lisse distale (fig. 10). Chez les plus grands individus, on trouve jusqu'à 3 tiges distales et une soie exopodiale marginale externe (e) (fig. 11).

Pléopodes II ỗ: Protopodite subquadrangulaire, presque carré, portant 3 longues tiges plumeuses sur la marge interne (fig. 12). Exopodite bi-articulé presque aussi long que l'endopodite chez les plus gros $\delta$. L'article distal est long et falciforme, courbé en direction externe. Il porte 3-5 courtes tiges lisses sur sa marge distale et distale-externe (fig. 13). Sa marge interne comporte une aire pilifère importante (fig. 12 et 13). Chez les jeunes $\hat{\delta}$ adultes, cet exopodite est encore plus court que l'endopodite et beaucoup moins arqué. Endopodite cylindroïde long et étroit, légèrement incurvé vers l'extérieur. Son aspect général est très caractéristique du genre Proasellus: partie proximale sans apophyses, mais avec un talon interne aplati; extrémité portant une apophyse tergale à direction externe; goulot plus court que l'apophyse et de faible diamètre, à fente sternale-externe visible, prolongée proximalement par l'orifice afférent de la vésicule interne largement ouvert (fig. 14).

Pléopodes III: Exopodite (opercule) à suture médiane non oblique, presque glabre (fig. 15). Endopodite charnu et respiratoire très petit.

Pléopodes IV: Exopodite ovalaire très grand avec area charnue très développée. Seule la linea area est visible. Endopodite charnu et respiratoire pratiquement aussi long, mais plus étroit que l'exopodite (fig. 16).

Pléopodes V: Exopodite et endopodite ovalaires subégaux. Linea duplex seule bien visible sur Pexopodite. Parfois une petite portion externe de la linea articularis apparaît. Endopodite charnu respiratoire (fig. 17).

Uropodes: Beaucoup plus courts que le pléotelson, ils sont fragiles et manquent sur la plupart des individus. Exopodite un peu plus court que l'endopodite (fig. 18). 

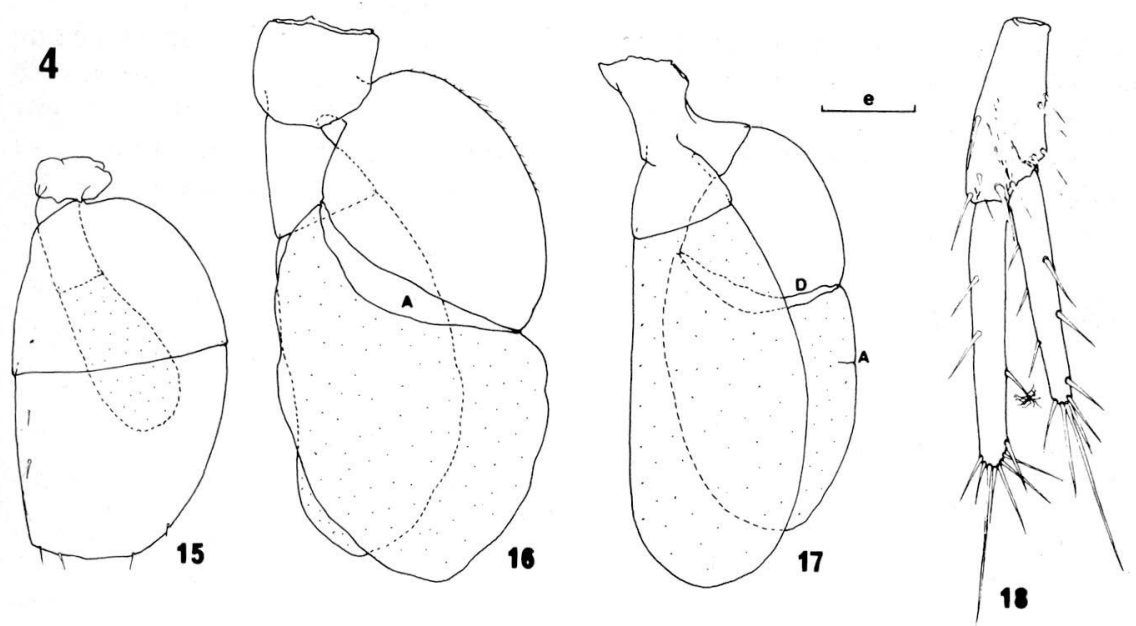

Fig. 15. Pléopode III gauche de Pallotype, face sternale, $\mathrm{e}=200 \mu$.

Fig. 16. Pléopode IV gauche de la même, face sternale, $\mathrm{e}=100 \mu$.

Fig. 17. Pléopode $\mathrm{V}$ droit, face tergale, du type, $\mathrm{e}=100 \mu$.

Fig. 18. Uropode droit, en vue tergale, d'un individu de $3,3 \mathrm{~mm}$ environ, de la station 3 , e = $100 \mu$.

Affinités: Par la structure de ses différents appendices caractéristiques, $P$. lescherae n.sp. se montre nettement apparenté aux autres espèces souterraines de Proasellus de la région pyrénéo-cantabrique, constituant une faune que nous avons qualifiée d'ibéro-aquitaine (Magniez et Henry, 1969, p. 408), lignée qui s'est particulièrement diversifiée dans les eaux souterraines karstiques du pays basque, tant au Sud qu'au Nord des Pyrénées et dont l'espèce la plus anciennement connue est Proasellus spelaeus (Racovitza, 1922) installé dans des grottes du haut bassin du Gave de Mauléon (Pyrénées-Atlantiques, France). Mais, contrairement à la plupart des espèces de cette lignée, qui vivent dans les eaux karstiques libres, $P$. lescherae $n$.sp. montre des caractères d'adaptation à la vie interstitielle très nets: importante réduction de la taille des adultes, forte diminution de la prolificité, simplification de la chétotaxie du corps et des appendices. Comme pour les autres Asellides, nous constatons cependant que cette adaptation ne modifie sensiblement, ni la taille des oeufs, ni la taille des jeunes stades larvaires libres, qui restent du même ordre que celles des espèces épigées ou des espèces souterraines de grande taille.

\section{RESUME}

Proasellus lescherae n.sp. est une petite espèce interstitielle qui vit dans le sousécoulement du bassin du Rio Guadalope (affluent de l'Ebre). Il est apparenté 
aux Aselles des eaux souterraines de la région pyrénéo-cantabrique (lignée de Proasellus spelaeus Racovitza). La taille moyenne des femelles en intermue de reproduction est faible $(2,9 \mathrm{~mm})$ et la prolificité est réduite (6 à 10 oeufs par portée). Pourtant, la taille des oeufs et des stades larvaires intramarsupiaux est identique à celles des espèces épigées et hypogées du genre qui atteignent des tailles plus grandes au stade adulte.

Manuscrit terminé en octobre 1977.

\section{BIBLIOGRAPHIE}

HENRY, J. P., 1976: Recherches sur les Asellidae hypogés de la lignée cavaticus (Crustacea, Isopoda, A sellota). Th. Sci. Nat. Univ. Dijon, No. de dépôt C.N.R.S.: 12 143, 270 pp.

HENRY, J. P.\& MAGNIEZ, G., 1970: Contribution à la systématique des Asellides. Ann. Spéléol. France, 23, 335-367.

Les Asellides (Crustacea, Isopoda, Asellota) des eaux souterraines d'Espagne et leur répartition. Actes du 6ème Symposium de Spéléologie, Biospéléologie, Terrassa (Barcelone), 1977, 29-36.

(1978) In ILLIES, J. Limnofauna Europaea, Isopoda, (2ème édition).

MAGNIEZ, G., 1975: Les stations de Stenasellus virei Dollfus, Crustacé Isopode troglobie (suite). Sous le Plancher, Organe du Spéléo-Club de Dijon, N.S., 12, 2, 22-26, (1973).

MAGNIEZ, G. \& HENRY, J. P., 1969: Un nouvel Aselle hypogé de France: Proasellus vandeli n.sp. Remarques sur les Proasellus ibéro-aquitains. Ann-Spéléol. France, 24, 399-411. 